EPJ manuscript No.

(will be inserted by the editor)

\title{
Limit distributions of scale-invariant probabilistic models of correlated random variables with the $q$-Gaussian as an explicit example
}

\author{
Rudolf Hanel ${ }^{1}$, Stefan Thurner ${ }^{1,3}$, and Constantino Tsallis ${ }^{2,3}$ \\ ${ }^{1}$ Complex Systems Research Group, HNO, Medical University of Vienna, Währinger Gürtel 18-20, A-1090, Austria \\ 2 Centro Brasileiro de Pesquisas Físicas, Rua Xavier Sigaud 150, 22290-180 Rio de Janeiro-RJ, Brazil \\ ${ }^{3}$ Santa Fe Institute, 1399 Hyde Park Road, Santa Fe, NM 87501, USA
}

the date of receipt and acceptance should be inserted later

\begin{abstract}
Extremization of the Boltzmann-Gibbs (BG) entropy $S_{B G}=-k \int d x p(x) \ln p(x)$ under appropriate norm and width constraints yields the Gaussian distribution $p_{G}(x) \propto e^{-\beta x^{2}}$. Also, the basic solutions of the standard Fokker-Planck (FP) equation (related to the Langevin equation with additive noise), as well as the Central Limit Theorem attractors, are Gaussians. The simplest stochastic model with such features is $N \rightarrow \infty$ independent binary random variables, as first proved by de Moivre and Laplace. What happens for strongly correlated random variables? Such correlations are often present in physical situations as e.g. systems with long range interactions or memory. Frequently $q$-Gaussians, $p_{q}(x) \propto\left[1-(1-q) \beta x^{2}\right]^{1 /(1-q)}\left[p_{1}(x)=p_{G}(x)\right]$ become observed. This is typically so if the Langevin equation includes multiplicative noise, or the FP equation to be nonlinear. Scale-invariance, i.e. exchangeable binary stochastic processes, allow a systematical analysis of the relation between correlations and nonGaussian distributions. In particular, a generalized stochastic model yielding $q$-Gaussians for all $(q \neq 1)$ was missing. This is achieved here by using the Laplace-de Finetti representation theorem, which embodies strict scale-invariance of interchangeable random variables. We demonstrate that strict scale invariance together with $q$-Gaussianity mandates the associated extensive entropy to be BG.
\end{abstract}

PACS. 05.20.-y Classical statistical mechanics - 02.50.Cw Probability theory $-05.90 .+\mathrm{m}$ Other topics in statistical physics, thermodynamics, and nonlin. dyn. systems - 05.70.-a Thermodynamics

One of the cornerstones of statistical mechanics is the functional connection of the thermodynamic entropy with the set of probabilities $\left\{p_{i}\right\}$ of microscopic configurations. For the celebrated Boltzmann-Gibbs (BG) theory, this functional is given by

$$
S_{B G}=-k \sum_{i=1}^{N} p_{i} \ln p_{i},
$$

where $N$ is the total number of microscopic states which are compatible with the information available about the system. This powerful connection is in principle applicable to a vast class of relevant systems, including (classical) dynamical ones whose maximal Lyapunov exponent is positive warranting strong chaos, hence mixing in phase space, hence ergodicity (Boltzmann, in some sense, embodied all these features in his molecular chaos hypothesis). Within this theory exponential distributions, i.e. exponential statistical factors, emerge naturally.

In particular, the Gaussian distribution $p_{G} \propto e^{-\beta x^{2}}$ with $\beta>0$ is found to be the (i) velocity (Maxwell) distribution of any classical many-body Hamiltonian system in thermal equilibrium with a thermostat i.e., if the interactions between its elements are sufficiently short-ranged, or inexistent. Furthermore, this important probabilistic form (ii) maximizes the (continuous version of the) entropy $S_{B G}=-\int d x p(x) \ln [p(x)]$ under the basic constraints of normalizability and finite width; (ii) constitutes the exact solution of the simplest form of the (linear) Fokker-Planck equation (i.e. corresponding Langevin equations with purely additive noise); (iii) is the $N \rightarrow \infty$ attractor of the appropriately centered and scaled sum of $N$ independent (or weakly correlated in an appropriate sense) discrete or continuous random variables whose second moment is finite (Central Limit Theorem). The simplest probabilistic model which realizes these paradigmatic features is a set of $N$ independent equal binary random variables (each of them taking, say the values 0 and 1 , with probability $1 / 2$ ). The probability of having, for fixed $N, n 1$ 's is given by $\frac{N !}{n !(N-n) !} 2^{-N}$. Its limiting distribution is, after centering and scaling, a Gaussian (as first proved by de Moivre and Laplace), and its (extensive) entropy is the one of $B G$, since $S_{B G}(N)=N k \ln 2$. 
Rudolf Hanel et al.: Title Suppressed Due to Excessive Length

What happens with the above properties when these binary random variables are not independent and the correlations between those variables (homogeneously spread over the system) are strong enough? There is in principle no reason for expecting the relevant limiting distribution to be a Gaussian, and the entropy which is extensive (i.e., $S(N) \propto N$ for $N \gg 1$ ) to be $S_{B G}$. The purpose of this paper is to answer such questions for a mathematically intriguing class of correlated processes - which can be interpreted as prototypical mean field models - and therefore are relevant in natural, artificial and even social systems. Let us discuss the frequently occurring $q$-Gaussians, a natural generalization of the Gaussians, defined as

$$
p_{q}(x) \propto\left[1-(1-q) \beta x^{2}\right]^{1 /(1-q)} \quad\left[p_{1}(x)=p_{G}(x)\right],
$$

where $x \in R$ for $q<3$ (for $q \geq 3$, normalizability is lost), and $x^{2} \leq 1 /[(1-q) \beta]$ for $q<1$. (i) $q$-Gaussians appear, e.g., as the exact solutions of paradigmatic nonMarkovian Langevin processes [1,2] which lead to inhomogeneous linear [3], or homogeneous nonlinear 4,5] FokkerPlanck equations. (ii) $q$-CLT attractors are $q$-Gaussians 6]. (iii) The extremization of $S_{q}$ with norm and finite width constraints yields $q$-Gaussians. $S_{q}$ [7] is a (generically nonadditive [8]) generalization of BG entropy, namely

$$
S_{q}=k \frac{1-\int d x[p(x)]^{q}}{q-1} \quad\left(q \in R ; S_{1}=S_{B G}\right) .
$$

Usually the extensive entropy of systems is given by the Boltzmann Gibbs entropy $S_{B G}$ which is identical with $S_{q}$ for $q=1$. It is noteworthy at this point that there exist systems with distribution functions (which are in general not q-Gaussian) depending on the system size $N$ in such a way that $S_{B G}$ is not the extensive entropy any more while $S_{q}$ becomes the extensive entropy for specific values of $q<1$ denoted by $q_{\text {ent }}$, i.e., $S_{q_{\text {ent }}}(N) \propto N(N \gg 1),[9,10$. As is well known, for all standard short-range-interacting many-body Hamiltonian systems, we have $q_{\mathrm{ent}}=1$, i.e. $S_{B G}$, which is identical to $S_{1}$, is extensive. (iv) Numerical indications [11] for the distributions of velocities in quasistationary states of long-range Hamiltonians 12 suggest $q$-Gaussians. Different interpretations of the situation are given in 13,14. (v) Further, experimental and observational evidence for $q$-Gaussians exists for the motion of biological cells [15,16], defect turbulence [17, solar wind [18, 19], cold atoms in dissipative optical lattices [20, dusty plasma 21, among others. Numerical indications are also available at the edge of chaos of unimodal maps 22. In [24] a specific model for correlated binary random variable was shown to converge to $q$-Gaussian distributions in the thermodynamic limit for $q<1$. Yet, no mechanism was given of how such models can be generated from general principles. This will be done in this paper. In particular, it will be shown how the model given in [24] can be generated and how by the same means $q$-Gaussian models can be generated for $1<q<3$.

In the following we consider binary exchangeable stochastic processes, with correlated elements say from $x \in$ $\{0,1\}$. Exchangeable means that the $N$-point probabilities
$p_{N}\left(x_{1}, x_{2}, \ldots, x_{N}\right)$ of the process are totally symmetric in its arguments for all $N$ and that $p_{N}$ can always be obtained by marginalization of $p_{N+1}$. In particular, the probability of a specific sequence $\left(x_{1}, x_{2}, \ldots, x_{N}\right)$, a microstate, does not depend on the order of binary events, but only on the number $n$ of events in the state $x_{i}=0$ and $N-n$ events in the state $x_{j}=1$. Following the notation in [24] we denote this probability as $r_{n}^{N}$. There are $\left(\begin{array}{l}N \\ n\end{array}\right)$ such micro-states (where the order of the $x_{i}$ is exchanged), and the probability of finding any situation with $n$ events in one state is given by

$$
p_{n}^{N}=\left(\begin{array}{c}
N \\
n
\end{array}\right) r_{n}^{N} \quad, \quad \sum_{n=0}^{N} p_{n}^{N}=1
$$

Total symmetry of the micro-states, $\forall N$, implies $r_{n}^{N-1}=$ $r_{n}^{N}+r_{n+1}^{N}$, sometimes referred to as the Leibniz triangle rule [9], or scale-invariance of the distribution. Scaleinvariance means here that at every 'scale' $N$ the relation $p_{N}\left(x_{1}, x_{2}, \ldots, x_{N}\right)=\sum_{x_{N+1}} p_{N+1}\left(x_{1}, x_{2}, \ldots, x_{N+1}\right)$ holds (where the sum runs over the possible discrete values of the random variable), i.e. the distribution at a lower scale can always be obtained by marginalization of higher scales. What does this mean for physical systems? If we look at the binary stochastic process in terms of coin tosses, this means that the transition probabilities that bias the $N$ 'th coin toss depend only on the number of tails and heads thrown after $N-1$ tosses and not on the particular history of coin tosses. If the coin tosses steer a random walk this means that the next step of the random walk is biased by the elapsed time (number of coin tosses $N$ ) and the distance the random walk has covered to its origin $(N-2 n)$ but not on the particular path the random walk has taken. In all Hamiltonian systems where the interactions are sufficiently long-range and strong enough for mean field solutions to be exact or close to exact totally symmetric correlations become physically relevant. Clearly, when mean field solutions become exact correlations between the state variables become totally symmetric. For binary state variables, i.e. Ising like spin systems, this implies that the conditional probability of one arbitrary spin being in one particular state conditioned on the state of all other spins only depends on the number of spins in one or the other state and not on the particular spin configuration of the system. Correlations introduced by such interactions can be understood in terms of exchangeable processes.

For dealing with such binary correlated systems the following representation was suggested by Laplace in 1774 , and later rediscovered by de Finetti [25, 26];

$$
r_{n}^{N}=\int_{0}^{1} d y y^{n}(1-y)^{N-n} g(y) \quad\left[\int_{0}^{1} d y g(y)=1\right] .
$$

This representation ensures Leibniz triangle rule and normalization, $\sum_{n=0}^{N}\left(\begin{array}{l}N \\ n\end{array}\right) r_{n}^{N}=1$. Note that the non-negative $g$ introduces the correlations in the stochastic process. In the case of independent variables, we have $g(y)=\delta(y-p)$, i.e. $r_{n}^{N}=p^{n}(1-p)^{N-n}$ is the usual binomial factor for 
independent trials. The fact that any stochastic process which is exchangeable to all scales $N$ can be represented in this way is called the Laplace-de Finetti theorem 25. 27,26 (if processes are exchangeable only up to a scales $N_{\max }$ positivity of $g$ is not ensured and negative correlations can occur [26]).

In order to pass from probabilities to distributions and from sums to integrals in a Riemann-Stieltjes sense in the thermodynamic limit $N \rightarrow \infty$ we use the well known properties of the Beta function, $B(a, b) \equiv \int_{0}^{1} d x x^{a-1}(1-x)^{b-1}$, to re-express the binomial factor in Eq. (4). Defining $\rho_{n}^{N} \equiv$ $(N+1) p_{n}^{N}$ turns Eq. (4) into

$$
\rho_{n}^{N}=\frac{\int_{0}^{1} d x x^{n}(1-x)^{N-n} g(x)}{\int_{0}^{1} d x x^{n}(1-x)^{N-n}}, \quad 1=\sum_{n=0}^{N} \frac{1}{N+1} \rho_{n}^{N} .
$$

As a consequence of this construction the natural domain of the limit distribution $\rho(y)$ is the interval $y \in[0,1]$. Using $y_{n}^{N}=(1 / 2+n) /(N+1)$, for $n=0,1, \ldots, N$, as discretization of $[0,1]$ in the limit one gets $1 /(N+1) \rightarrow d y$ and $y_{n}^{N} \rightarrow y$. Denote the natural number closest to the value $(N+1) y-1 / 2$ by $[(N+1) y]$ then the limit distribution gets $\rho(y)=\lim _{N \rightarrow \infty} \rho_{[(N+1) y]}^{N}$. These arguments imediately lead to

$$
\rho(y)=\lim _{N \rightarrow \infty} \frac{\int_{0}^{1} d x\left[x^{y}(1-x)^{1-y}\right]^{N} g(x)}{\int_{0}^{1} d x\left[x^{y}(1-x)^{1-y}\right]^{N}} .
$$

Notice that the maximum of $x^{y}(1-x)^{1-y}$ with respect to $x$ is obtained at $x=y$ and gets amplified above all other values of the function by the power $N$. For $N \rightarrow \infty$ we therefore get a delta sequence and

$$
\delta(x-y)=\lim _{N \rightarrow \infty} \frac{\left[x^{y}(1-x)^{1-y}\right]^{N}}{\int_{0}^{1} d x\left[x^{y}(1-x)^{1-y}\right]^{N}} .
$$

Consequently,

$$
\rho(y)=\int_{0}^{1} d x \delta(x-y) g(x)=g(y)
$$

i.e. $\lim _{N \rightarrow \infty} \sum_{n}\left|\rho_{n}^{N}-g\left(y_{n}^{N}\right)\right|=0$. The limit distribution $\rho$ and the function $g$ generating the Leibniz-triangle $r_{n}^{N}$ are in fact identical! Therefore, once a desired limiting distribution on $[0,1]$ is given, one can simply write down the sequence of $r_{n}^{N}$ which is generating it! Though it is known that $g$ may become negative [26], if scale-invariance is broken and the model is exchangeable only up to a maximal scale $N_{\max }$, this interpretation of $g$ makes it clear why non-negativity of $g$ is required in the limit $N \rightarrow \infty$. The limit distribution $\rho(y)$, i.e. $g(y)$, of binary exchangeable processes is defined on $y \in[0,1]$, where $y$ is basically the ratio of events $n / N$ in the limit of large $N$. However, prototypical processes, e.g. spin-systems or random walks, depend on binary processes (spins up/down or number of left/right steps). Yet, their associated observable variable is not the ratio $n / N$ of binary events but some other descriptive variable $z$ (the magnetization or the position of a random walk). Since the domain of the descriptive variable $z$ will in general not be $[0,1]$ (e.g. typically $[-1,1]$ for spin systems and $[-\infty, \infty]$ for random walks) it has to be clear from the beginning that the form of the limit distribution of the descriptive variable $z$ will not only depend on $g$ but also on the relation between $z$ and the binary process, i.e. on $y=n / N$, which may or may not depend on the scale $N$. E.g., if equidistant spacing on intervals $\left[-R_{N}, R_{N}\right] \subset[-\infty, \infty]$ as $R_{N} \rightarrow \infty$ is considered (as is the case of CLT) this relation will in general be a variable transformation depending on the scale $N$. For simplicity we only consider the case of $N$ independent transformations of variables between $y$ and $z$. We assume a symmetric distribution $G(z)$ of the descriptive variable $z$ that can be obtained by a transformation of variables defined by $d z G(z)=d y \rho(y)$. We will call a strictly monotonous increasing antisymmetric functions $f$, such that the effective stochastic variable $z$ will take the values $z_{n}^{N}=f\left(2 y_{n}^{N}-1\right)$, a symmetric representation of the binary process. To be clear, $z=f(2 y-1)$ is precisely the change of variables relating the generating function $g$ of the binary process with the limit distribution $G$ of the observable process $z$. In particular, $G$ and $g$ are one-to-one related by $2 f^{\prime}(2 y-1) G(f(2 y-1))=g(y)$. Each pair $(f, g)$ exactly defines the distribution $G$ of the binary process. Moreover, fixing $G$ and the representation $f$ uniquely determines $g$. Inversely, for a given observable distribution $G$, any pair $(f, g)$ that represents $G$ can serve as a stochastic model for $G$. A physical view on the meaning of $f$ and $g$ can be given by Galton's board, where $f$ corresponds to the positions of needles on the board while $g$ fixes the probabilities of balls being reflected to the left or right at the condition of hitting some needle.

We now proceed to derive a stochastic model with $q$-Gaussian limit distributions. For the case of $q<1$, the $q$-Gaussian $G_{q}(z)=\frac{1}{Z_{q}}\left[1-(1-q) z^{2}\right]^{1 / 1-q}$ is defined on a compact support, $|z| \leq \frac{1}{\sqrt{1-q}}$. To identify it with the limiting distribution we have to map $z$ to the unit interval by $z=f(2 y-1)=\frac{1}{\sqrt{1-q}}(2 y-1)$. Since the support of the $q$-Gaussian $G_{q}(z)$ for $q<1$ is compact an affine transformation is the natural choice for the change of variables. Under an affine variable transformation $G_{q}(z) \rightarrow \rho(y)=\frac{1}{Z_{q}} 4 y^{\frac{1}{1-q}}(1-y)^{\frac{1}{1-q}}$, where $Z_{q}=4 B\left(\frac{1}{1-q}+1, \frac{1}{1-q}+1\right)$. Consequently, by introducing the notation $\nu \equiv \frac{1}{1-q}+1$, we get $g(y)=\frac{[y(1-y)]^{\frac{1}{1-q}}}{B(\nu, \nu)}$ and, finally, by using Eq. (5),

$$
r_{n}^{N}(q)=\frac{B(\nu+n, \nu+N-n)}{B(\nu, \nu)} .
$$

To retrieve the original $q$-Gaussian $G_{q}(z)$ one has to perform the inverse coordinate transformation. This maps the discretization

$$
z_{n}^{N}=f\left(2 y_{n}^{N}-1\right), \quad \text { i.e., } \quad z_{n}^{N}=\left(2 y_{n}^{N}-1\right) / \sqrt{1-q}
$$

and the discretization-width becomes $d z \equiv 2 /(\sqrt{1-q}(N+$ $1))$, which takes into account the the factor $2 f^{\prime}\left(2 y_{n}^{N}-\right.$ 
1) $=2 / \sqrt{1-q}$ from the change of variables. Analogous to $\rho$ the discrete formulation of the $q$-Gaussian $G_{q}$ reads $F_{n}^{N}=(d z)^{-1}\left(\begin{array}{c}N \\ n\end{array}\right) r_{n}^{N}$ and

$$
\lim _{N \rightarrow \infty} \sum_{n}\left|F_{n}^{N}-G_{q}\left(z_{n}^{N}\right)\right| \rightarrow 0
$$

This is exactly the model which was heuristically found in 24. There the model was presented as

$$
r_{0}^{N}(q)=\frac{(N+\nu-1)(N+\nu-2) \ldots \nu}{(N+2 \nu-1)(N+2 \nu-2) \ldots(2 \nu)},
$$

for integer values of $\nu$, which is obviously a particular case of Eq. (10).

Since the $q$-Gaussian has no compact support for $q>1$ the situation becomes more involved, since now one has to map the real axis to the unit interval $[0,1]$. Such a $q$ Gaussian $G_{q}(z)$ with $z \in[-\infty, \infty]$ may be thought of as distributions of the distance $z$ a peculiar random walk has covered in the long time limit. There is no map $f$ now that is a natural choice, a priori. However, in order to explicitly compute the probabilities $r_{n}^{N}$ one may choose a map such that $r_{n}^{N}$ is given in terms of Beta functions, as before. This leads to the situation that pairs of $q$-Gaussians, one with $q<1$ and another with $q>1$, can be generated by the same binary process and differ only in terms of the representation $f$.

Let us find a map $f$ such that $f(2 y-1)$ again maps $y \in$ $[0,1]$ to $z \in[-\infty, \infty]$. Using the normalization condition of the $q$-Gaussian this variable transformation implies $1=$ $\int_{-\infty}^{\infty} d z G_{q}(z)=\int_{-1}^{1} d x f^{\prime}(x) G_{q}(f(x))$ and identify

$$
g(y)=2 f^{\prime}(2 y-1) G_{q}(f(2 y-1)) .
$$

A particular $f$ to compute $r_{n}^{N}$ in closed form is given by

$$
f(y)=\frac{y}{\sqrt{1-y^{2}}} \frac{1}{\sqrt{q-1}} \quad \text { and } \quad f^{\prime}(y)=\frac{\left(1-y^{2}\right)^{-3 / 2}}{\sqrt{q-1}} .
$$

Noting, that this model $f$ for the $q$-Gaussian implies $[1-$ $\left.(1-q) f(y)^{2}\right]^{1 /(1-q)}=\left(1-y^{2}\right)^{1 /(q-1)}$. Inserting this into Eq. (14) we finally get the stochastic model,

$$
r_{n}^{N}(q)=\frac{B(\mu+n, \mu+N-n)}{B(\mu, \mu)},
$$

where we have used the notation $\mu=\frac{1}{q-1}-\frac{1}{2}$. All that is left is to place everything correctly. We use the same discretization $y_{n}^{N}=(1 / 2+n) /(N+1)$ for the interval $[0,1]$ as for $q<1$. Again, $d y_{n}^{N}=1 /(N+1)$ is the width of the discretization on $[0,1]$ and with $\rho_{n}^{N}=\left(d y_{n}^{N}\right)^{-1}\left(\begin{array}{l}N \\ n\end{array}\right) r_{n}^{N}$, we get that $\lim _{N \rightarrow \infty} \sum_{n}\left|\rho_{n}^{N}-g\left(y_{n}^{N}\right)\right|=0$, as a power of $N$ as in the $0<q<1$ case. To retrieve the $q$-Gaussian $G_{q}(z)$, one has to perform the inverse coordinate transformation $y \in[0,1] \rightarrow z \in[-\infty, \infty]$. This is a little more complicated since the discretization width now depends on $N$ and $n$. In particular, mapping back to $[-\infty, \infty]$ gives the discretization $z_{n}^{N}=f\left(2 y_{n}^{N}-1\right)$, i.e. $z_{n}^{N}=\left(y_{n}^{N}-\right.$
$1 / 2) / \sqrt{(q-1) y_{n}^{N}\left(1-y_{n}^{N}\right)}$, and the discretization width becomes $d z_{n}^{N} \equiv\left(y_{n}^{N}\left(1-y_{n}^{N}\right)\right)^{-3 / 2} /(4(N+1) \sqrt{q-1})$. The discretized version of the $q$-Gaussian for $q>1$ now reads, $F_{n}^{N}=\left(d z_{n}^{N}\right)^{-1}\left(\begin{array}{c}N \\ n\end{array}\right) r_{n}^{N}$. In the limit we get $\lim _{N \rightarrow \infty} \sum_{n} \mid F_{n}^{N}-$ $G_{q}\left(z_{n}^{N}\right) \mid \rightarrow 0$, as a power of $N$. Comparing Eq. (10) and Eq. (16) it is obvious that for the two models of the $q$ Gaussian distribution $(q<1$ and $\bar{q}>1)$ the generating binary processes $r_{n}^{N}$ are identical whenever $\nu=\mu$. $\nu$ and $\mu$ are functions of $q$ and $\bar{q}$, and $\nu(q)=\mu(\bar{q})$ establishes a relation between two $q$-Gaussian distributions generated by an identical exchangeable binary stochastic process, i.e. $\bar{q}=\frac{7-5 q}{5-3 q} ; q$ increasing from $-\infty$ to 1 yields $\bar{q}$ decreasing from $5 / 3$ to 1 . Therefore, the models of $q$ Gaussian distributions with $q<1$ are conjugate with the model of $\bar{q}$-Gaussian with $\bar{q} \in[1,5 / 3]$ in the sense of being driven by an identical binary stochastic process. The class of $\bar{q}$-Gaussian distributions with $\bar{q} \in(5 / 3,3]$, which is exactly the class of normalizable $\bar{q}$-Gaussian distributions with diverging second moment, are not identified with a $q<1$. The corresponding binary processes are unique in this sense. For instance, choosing $1<\bar{q}=2$ requires $1>q=3$, which is impossible. For $\bar{q}=2$ it follows that $\mu=1 / 2$ and the associated binary process $r_{n}^{N}=B(1 / 2+n, 1 / 2+N-n) / B(1 / 2,1 / 2)$ has no representation for any $q<1$, and $r_{0}^{N}=\frac{B(1 / 2,1 / 2+N)}{B(1 / 2,1 / 2)}=$ $2^{-2 N}\left(\begin{array}{c}2 N \\ N\end{array}\right)$. Of course one can choose many families $\left(f_{q}, g_{q}\right)$ of models for $q$-Gaussian limit distributions and, for each strictly monotonous function $\bar{q}:[-\infty, 1] \mapsto[1,3]$, it is possible to construct conjugate families of models in the sense that they are generated by the same family of binary processes. In fact when the function $L_{q}$ is defined by $\int_{0}^{L_{q}(z)} d z^{\prime} G_{q}\left(z^{\prime}\right)=\int_{0}^{z} d z^{\prime \prime} G_{\bar{q}(q)}\left(z^{\prime \prime}\right)$ and $\left(f_{q}, g_{q}\right)$ is a model for a $q$-Gaussian with $q<0$ then $\left(L_{q}\left(f_{q}\right), g_{q}\right)$ is a conjugate model of the $\bar{q}$-Gaussian with $\bar{q}=\bar{q}(q)$. This allows to conjugate families of models for different dualities recognized in $q$-statistics, e.g. $\bar{q}=(5-3 q) /(3-q)$ for $q$-Fourier transforms [28.

We have explicitly derived two possible stochastic models for correlated and exchangeable binary random variables, which lead to exact $q$-Gaussians as the limiting distributions, while strictly satisfying Leibniz rule,

$$
r_{n}^{N}= \begin{cases}\frac{B\left(\frac{2-q}{1-q}+n, \frac{2-q}{1-q}+N-n\right)}{B\left(\frac{2-q}{1-q}, \frac{2-q}{1-q}\right)} \quad \text { if } \quad q<1, \\ \frac{1 / 2^{N}}{B\left(\frac{3-q}{2 q-2}+n, \frac{3-q}{2 q-2}+N-n\right)} & \text { if } \quad q=1, \\ \frac{B\left(\frac{3-q}{2 q-2}, \frac{3-q}{2 q-2}\right)}{2} & \text { if } \quad 1<q<3,\end{cases}
$$

$q=1$ can be obtained by both $q \rightarrow 1^{ \pm}$. Eq. (17) follows for the affine representation for $q<1$ and for the representation $f$ given in Eq. (15) for $q>1$. For different families of representations $f_{q}$ of the q-Gaussians the equation has to be adapted accordingly.

Lately the question whether $S_{Q}$ can be the extensive entropy for specific systems for $Q<1$ has been discussed, e.g. in [9,10]. With the Laplace-de Finetti representation 


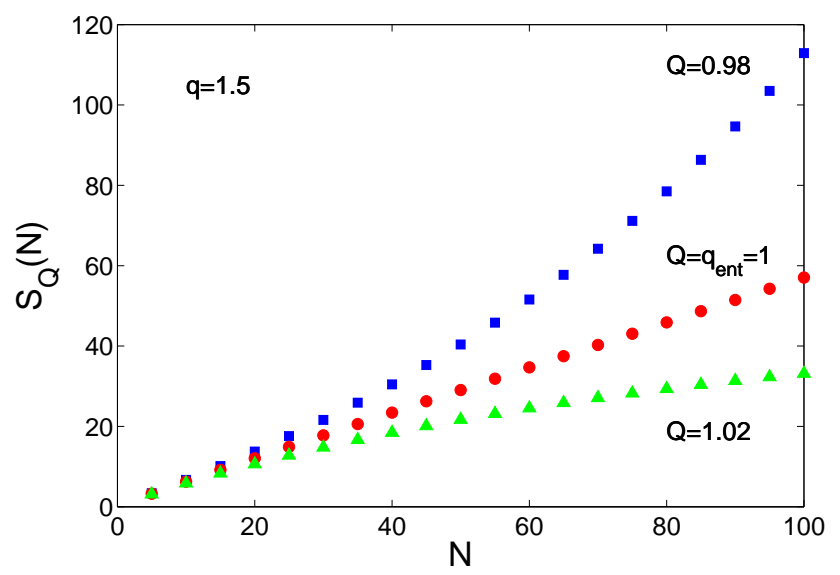

Fig. 1. Nonadditive entropy $S_{Q}$ vs. $N$ for a $q$-Gaussian with $q=1.5$, for $Q=0.92,1$ and 1.02. Clearly, only $Q=1$ is extensive (i.e., $S_{1}(N) \propto N$, for $N \gg 1$ ).

we consider the entropy $S_{Q}$ for large $N$ and prove that the value of $Q$ for which $S_{Q}$ is extensive with respect to the distribution of binary states is $Q=1$, i.e. $q_{\text {ent }}=1$, by straight foreward calculation:

$$
\begin{aligned}
& S_{Q}[g]=\frac{1-\sum_{n=0}^{N}\left(\begin{array}{c}
N \\
n
\end{array}\right)\left[r_{n}^{N}\right]^{Q}}{Q-1} \\
= & \frac{\sum_{n=0}^{N} \frac{\left((N+1)\left(\begin{array}{c}
N \\
n
\end{array}\right)\right)^{1-Q}}{N+1}\left[\frac{\int_{0}^{1} d x x^{n}(1-x)^{N-n} g(x)}{\int_{0}^{1} d x x^{n}(1-x)^{N-n}}\right]^{Q}-1}{1-Q} \\
= & \frac{\int_{0}^{1} d y\left[\int_{0}^{1} d x\left[x^{y}(1-x)^{1-y}\right]^{N}\right]^{Q-1}[g(y)]^{Q}-1}{1-Q} .
\end{aligned}
$$

The first line follows from using the exchangeability-property together with the definition of the $q$-entropy. The second line can be obtained by inserting Eq. (5) for $r_{n}^{N}$ and using the properties of the $\beta$-function. The third line follows for large $N$, where $\sum_{n=0}^{N} 1 /(N+1) \rightarrow \int_{0}^{1} d y$, and using Eq. (8). To find the index $Q$ where $S_{Q}$ gets extensive requires $\frac{d S_{Q}[g]}{d N}$ to be a positive constant for $N \gg 1$ for some specific value of $Q$ which is called $q_{\mathrm{ent}}$. We find, by using Stirling's approximation,

$$
\frac{d S_{Q}[g](N)}{d N} \sim 2^{N(1-Q)} g(1 / 2) \ln 2 .
$$

Hence, for any $g(1 / 2)>0, S_{Q}(N)$ growing linearly with large $N$ requires that $Q=1$, since otherwise $S_{Q}(N)$ grows exponentially with $N$, i.e., $q_{\mathrm{ent}}=1$ for all such models. It should be noted that particular choices of representations $f$ and the observable process (distribution functions) only result in particular choices of $g$. Since the result is valid for all $g$ the result is basically independent of the particular choice of representing an exchangeable process.

We have shown that the Laplace-de Finetti representation is a suitable framework which allows to generate scale invariant probabilistic models by fixing their limit distribution and a map which describes how the domain of the observable variable of model is embedded on the real axis on each scale $N$. We have demonstrated this with the $q$-Gaussian distributions as an example. We have constructed scale invariant models for $q$-Gaussians for all $q<3$ and have shown how notions of conjugate families of processes can be constructed. We have shown that the Boltzmann Gibbs entropy is the extensive entropy for all stochastic processes generated by scale-invariant binary processes.

We acknowledge useful remarks by A. Rodriguez, V. Schwammle, E.P. Borges and E.M.F. Curado. R.H. and S.T. acknowledge great hospitality at CBPF and partial support by Faperj and $\mathrm{CNPq}$ (Brazilian agencies) and FWF Project P19132.

\section{References}

1. Anteneodo C. and Tsallis C., J. Math. Phys., 44 (2003) 5194 .

2. Fuentes M.A. and Caceres M.O., Phys. Lett. A, 372 (2008) 1236-1239.

3. Borland L., Phys. Lett. A, 245 (1998) 67.

4. Plastino A.R. and Plastino A., Physica A, 222 (1995) 347.

5. Tsallis C. and Bukman D.J., Phys. Rev. E, 54 (1996) R2197.

6. Umarov S., Tsallis C. and Steinberg S., Milan J. Math., 76 (2008) [DOI 10.1007/s00032-008-0087-y]; for a more pedagogical version, see Queiros S.M.D. and Tsallis C., AIP 965, 21 (New York, 2007).

7. Tsallis C., J. Stat. Phys., 52 (1988) 479; Introduction to Nonextensive Statistical Mechanics - Approaching a Complex World (Springer, New York, 2009), in press.

8. Penrose O., Foundations of Statistical Mechanics: A Deductive Treatment (Pergamon, Oxford, 1970), p. 167.

9. Tsallis C., Gell-Mann M. and Sato Y., Proc. Nat. Acad. Sci., 102 (2005) 15377.

10. Caruso F. and Tsallis C., Phys. Rev. E, 78 (2008) 021101.

11. Pluchino A., Rapisarda A. and Tsallis C., Eur. Phys. Lett., 80 (2007) 26002; Physica A, 387 (2008) 3121.

12. Anteneodo C. and Tsallis C., Phys. Rev. Lett., 80 (1998) 5313.

13. Yamaguchi Y., Bouchet G. and Dauxois T., JSTAT, (2007) P01020.

14. Bouchet G. and T. Dauxois T., Phys. Rev. E, 72 (2005) $045103(\mathrm{R})$.

15. Upadhyaya A., Rieu J.-P., Glazier J.A. and Sawada Y., Physica A, 293 (2001) 549.

16. Thurner S., Wick N., Hanel R., Sedivy R. and Huber L.A., Physica A, 320C (2003) 475-484.

17. Daniels K.E., Beck C. and Bodenschatz E., Physica D, 193 (2004) 208.

18. Burlaga L.F. and Vinas A.F., Physica A, 356 (2005) 375.

19. Burlaga L.F., Vinas A.F., Ness N.F. and Acuna M.H., Astrophys. J., 644 (2006) L83-L86.

20. Douglas P., Bergamini S. and Renzoni F., Phys. Rev. Lett., 96 (2006) 110601.

21. Liu B. and Goree J., Phys. Rev. Lett., 100 (2008) 055003.

22. Tirnakli U., Beck C. and Tsallis C., Phys. Rev. E, 75 (2007) 040106(R); 0802.1138 [cond-mat.stat-mech].

23. Grassberger P., arXiv:0809.1406 [cond-mat.stat-mech]. 
24. Rodriguez A., Schwammle V. and Tsallis C., JSTAT (2008) P09006.

25. de Finetti B., Atti della R. Academia Nazionale dei Lincei 6, Memorie, Classe di Scienze Fisiche, Mathematice e Naturale, 4 (1931) 251299; Heath D., Sudderth W., Am. Stat., 30 (1976) 188-189.

26. Jaynes E.T., in Bayesian Inference and Decision Techniques with Applications, Goel P.K. and Zellner A., eds. (North-Holland, Amsterdam, 1986), p. 31.

27. Hewitt E., Savage L.J., Trans. Am. Math. Soc., 80 (1955) 470-501.

28. Nelson K.P., Umarov S., arXiv:0811.3777v1. 